\title{
Generating Superpositions of Higher Order Bessel Beams
}

\author{
R. Vasilyeu ${ }^{1}$, A. Dudley ${ }^{2,3}$, N. Khilo ${ }^{1}$, and A. Forbes ${ }^{2,3}$ \\ ${ }^{1}$ B.I. Stepanov Institute of Physics of NAS of Belarus, Nezalezhnasti Ave., 68, 220072 Minsk, Belarus \\ r.vasilyev@dragon.bas-net.by andn.khilo@dragon.bas-net.by \\ ${ }^{2}$ School of Physics, University of KwaZulu-Natal, Private Bag X54001, Durban 4000, South Africa \\ ${ }^{3}$ CSIR National Laser Centre, PO Box 395, Pretoria 0001, South Africa \\ ADudley@csir.co.za and AForbes1@csir.co.za
}

\begin{abstract}
An experimental setup to generate a superposition of higher-order Bessel beams by means of a spatial light modulator and ring aperture is presented. The experimentally produced fields are in good agreement with those calculated theoretically. (C)2009 Optical Society of America OCIS codes: (140.3300) Laser Beam Shaping; (080.4865) Optical vortices.
\end{abstract}

\section{Introduction}

There is an extensive body of literature on generating either zero order or higher-order Bessel beams. Visually, the zero order Bessel beam possesses a bright central maximum, while the higher-orders have a dark central vortex, which propagates over an extended distance in a diffraction-free manner. Zero order Bessel beams can be generated by illuminating a ring slit aperture, placed in the back focal plane of a lens, with a plane wave ${ }^{[1]}$. Diffractive optical elements, such as axicons ${ }^{[2,3]}$, and computer generated holograms ${ }^{[4,5]}$, can be used to generate both zero and higherorder Bessel beams. However, in this report we have developed a mechanism for the generation of the superposition of higher-order Bessel beams ${ }^{[6]}$, which implements the previously mentioned technologies, namely a ring slit aperture and computer generated holograms.

\section{Theoretical Background}

In our optical setup a spatial light modulator (SLM) is used to generate a superposition of two higher-order Bessel beams. The incident Gaussian beam is transformed to a ring field, with radius $R$ and width $2 \Delta$, by a ring slit aperture. This ring field is projected onto an SLM, where an azimuthally varying phase is imparted to the angular spectrum of the final beam:

$$
\Phi(r, \varphi)= \begin{cases}\exp (\operatorname{im} \varphi) & R \geq r \geq(R-\Delta) \\ \exp (\operatorname{in} \varphi) & R \leq r \leq(R+\Delta)\end{cases}
$$

This angular spectrum is then transformed into a superposition of two Bessel beams of order $m$ and $n$ by a Fouriertransforming lens.

The resulting superposition is calculated numerically by the use of the Kirchoff-Huygens diffraction integral

$$
A_{m, n}(r, \varphi, z)=A_{m}(r, \varphi, z)+A_{n}(r, \varphi, z)
$$

where,

$$
\begin{gathered}
A_{m}(r, \varphi, z)=\frac{-i k_{0} i^{m}}{f} \exp (i m \varphi) \int_{(R-\Delta)}^{R} \exp \left[-\frac{r_{1}^{2}}{w^{2}}+\frac{i k_{0} r_{1}^{2}}{2 f}\left(1-\frac{z}{f}\right)\right] J_{m}\left(\frac{k_{0} r r_{1}}{f}\right) r_{1} d r_{1} \\
A_{n}(r, \varphi, z)=\frac{-i k_{0} i^{n}}{f} \exp (\operatorname{in} \varphi) \int_{R}^{(R+\Delta)} \exp \left[-\frac{r_{1}^{2}}{w^{2}}+\frac{i k_{0} r_{1}^{2}}{2 f}\left(1-\frac{z}{f}\right)\right] J_{n}\left(\frac{k_{0} r r_{1}}{f}\right) r_{1} d r_{1}
\end{gathered}
$$

\section{Experimental Results}

The experimentally produced fields (depicted in figure 1) are compared with the calculated fields. 
(a)

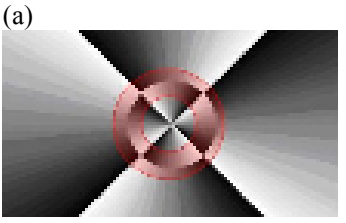

(e)

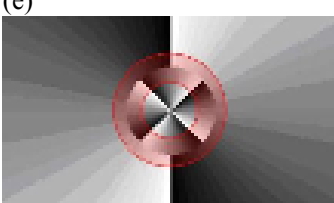

(i)

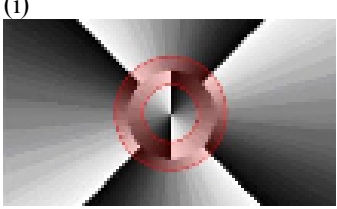

(b)

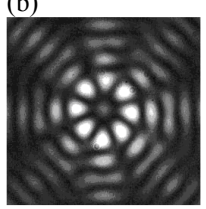

(f)

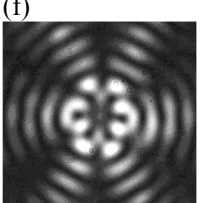

(j)

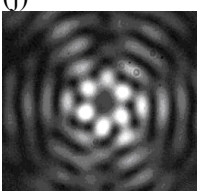

(c)

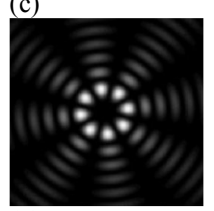

(g)

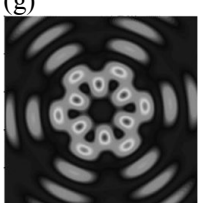

(k)

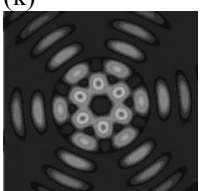

(d)

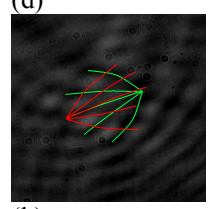

(h)

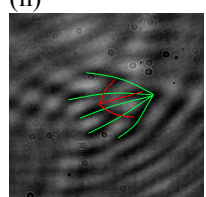

(1)

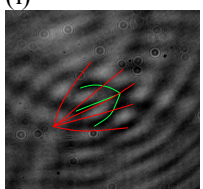

Fig. 1. The columns from left to right represent the phase pattern applied to the SLM, the observed intensity distribution of the superposition, the theoretical prediction, and interference pattern of the superposition field and a plane wave, respectively. Data is shown for (a) $-(\mathrm{d}): A_{4,-4},(\mathrm{e})-(\mathrm{h}): A_{2,-4}$, and (i) $-(1): A_{4,-2}$. The illuminated ring slit is shown as a red overlay on the phase patterns.

It is known that higher-order Bessel beams can be generated by the illumination of a ring slit aperture or axicon ${ }^{[3]}$ with a beam which has an azimuthal phase dependence, $\exp (\operatorname{im} \varphi)$, such as a Laguerre-Gauss beam. In this work, we have adapted this concept so that the light illuminating the ring slit aperture possesses two separable azimuthal phase components, namely $\exp (\operatorname{im} \varphi)$ and $\exp (\operatorname{in} \varphi)$, producing a superposition of an $\mathrm{m}^{\text {th }}$ order and $\mathrm{n}^{\text {th }}$ order Bessel beam. This is achieved by applying a phase pattern which consists of two separate parts (such as those represented in figure 1) to the liquid crystal display. The number of 'petals', present in the field, surrounding the vortex core is determined by the order of the two superimposed Bessel beams and is given as follows: $|\mathrm{m}|+|\mathrm{n}|$.

Due to the azimuthally varying phase which is imparted to the angular spectrum of the beam, the resulting fields possess vortices. The vortex structure of these fields is investigated by interfering these fields with a plane wave. It is known that fork-like patterns can be observed when a wavefront containing a screw dislocation is interfered with a plane wave. Some of our obtained experimental images, illustrating the fork-like patterns, are given in figure 1. From the images it is evident that two fork-like patterns are present in the interference pattern due to the two superimposed Bessel fields. The number of spaces between the fork 'prongs' is related to the number of screw dislocations present in the field and hence to the order of the Bessel beams present in the superposition.

Apart from investigating the field produced at the Fourier plane, the propagation of these fields was also investigated. It was noted that the intensity profile of the field rotates during the beams propagation which is in accordance with predicted results ${ }^{[7]}$.

\section{References}

[1] J. Durnin, J. J. Miceli, J. H. Eberly, “Diffraction-free beams,” Phys. Rev. Lett. 58, 1449 (1987).

[2] R. M. Herman, T. A. Wiggins, "Production and uses of diffractionless beams," J. Opt. Soc. Am. A 8, 932-942 (1991).

[3] J. Arlt and K. Dholakia, "Generation of high-order Bessel beams by use of an axicon," Opt. Comm. 177, 297-301 (2000).

[4] J. Turunen, A. Vasara, A. T. Friberg, "Holographic generation of diffraction-free beams," Appl. Opt. 27, 3959-3962 (1988).

[5] A. Vasara, J. Turunen, A. T. Friberg, "Realization of general nondiffracting beams with computer-generated holograms," J. Opt. Soc. Am. A 6, 1748-1754 (1989).

[6] R. Vasilyeu, A. Dudley, N. Khilo, A. Forbes, "Generating superpositions of higher order Bessel beams," To be submitted to Opt. Commun. (2009).

[7] P. A. Khilo, L. I. Kramoreva, and N. A. Khilo, “Optically controlled rotation of multibeam light field,” Proc. SPIE Vol. 4358, 250-253 (2001). 